\title{
Ambiphilic Allenes: Synthesis and Reactivity ${ }^{\dagger}$
}

\author{
David Tejedor, ${ }^{* a, b}$ Gabriela Méndez-Abt, ${ }^{a, b}$ Javier González-Platas, ${ }^{c}$ Miguel A. Ramírez ${ }^{d}$ Fernando \\ García-Tellado*a,b
}

Received (in $X X X, X X X)$ Xth $X X X X X X X X X 200 X$, Accepted Xth $X X X X X X X X X 200 X$

${ }_{5}$ First published on the web $X$ th $X X X X X X X X X 200 X$

DOI: 10.1039/b000000x

\begin{abstract}
Ambiphilic allenes are generated by an organocatalyzed domino reaction of alkyl propiolates and aromatic 1,2-diketones. In the absence of any external chemical agent, these allenes perform a 10 thermally-driven dimerization reaction to generate the corresponding fully-substituted cyclobutanes in a regio- and highly stereoselective manner.
\end{abstract}

The search for organic molecules expressing ambiphilic reactivity is a sought-after challenge in organic chemistry. ${ }^{1}$

15 Isocyanides constitute a paradigmatic example with a carbon atom able to react with both electrophiles and nucleophiles. ${ }^{2}$ Allenes armed with an e-donating group and an e-acceptor group at the $\mathrm{C} 3-\pi$ system (1,3-relationship) are expected to express this property at $\mathrm{C} 2$ position (Figure 1). Allene 20 reactivity is regulated to a large extent by the substitution pattern adorning the $\mathrm{C} 3-\pi$ system. ${ }^{3}$ On this sense, although the

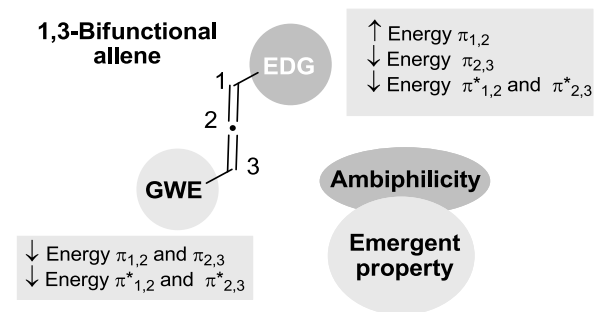

Figure 1. Substituent effect on the allene reactivity.

25

individual effect of e-donating (EDG) and e-withdrawing groups (EWG) on the allene reactivity are well established (Figure 1$),{ }^{3 a, 4}$ the overall effect when they operate in a 1,3assonant manner remains to be evaluated. With these 30 precedents we have initiated a research program aimed to the synthesis and the study of 1,3-bifunctional allenes as synthetically relevant ambiphilic reactants for the

${ }^{a}$ Instituto de Productos Naturales y Agrobiología, CSIC, Astrofísico

Francisco Sánchez 3, 38206 La Laguna, Tenerife, Spain.

fgarcia@ipna.csic.es; dtejedor@ipna.csic.es

${ }^{b}$ Instituto Canario de Investigación del Cáncer (www.icic.es)

${ }^{c}$ Servicio de Difracción de Rayos X, Departamento de Física

Fundamental II, Universidad de La Laguna, Astrofísico Francisco

Sánchez 2,38206 La Laguna, Tenerife, Spain.

${ }^{d}$ Instituto Universitario de Bioorgánica Antonio González,

Universidad de La Laguna, Astrofísico Francisco Sánchez 2,38206 La

Laguna, Tenerife, Spain.

$\dagger$ Electronic supplementary information (ESI) available: Experimental section and physical data for compounds 4-9. CCDC reference numbers 704023and 704024. For ESI and crystallographic data in CIF see DOI: $10.1039 / \mathrm{b} 000000 \mathrm{x}$ development of novel domino and multicomponent reactions. For experimental reasons related to our previous work on the 35 chemistry of non-metallic conjugated acetylides, ${ }^{5}$ we selected the allenic esters $\mathbf{1}$ as the target bifunctional allene for these studies. ${ }^{6}$

We envisioned that these allenic esters could be synthesized by a tertiary amine-catalyzed domino reaction 40 involving 1,2-diketones and terminal conjugated acetylides (Scheme 1). The manifold was devised on the basis of our previous studies on the organocatalyzed generation of conjugated acetylides $\mathbf{I}$ and their reactivity against different types of electrophiles. ${ }^{7}$ It was hypothesized that the ${ }_{45}$ intermediate II, obtained from the nucleophilic addition of $\mathbf{I}$ onto the 1,2-diketone, should suffer a rearrangement that would lead to the rupture of the more labile $\mathrm{CO}-\mathrm{CO}$ bond (BE $\approx 70 \mathrm{Kcal} / \mathrm{mol})$ via epoxide III. $^{8}$ Interestingly, and in accordance with our previous experiences, in the process to ${ }_{50}$ form the desired allene $\mathbf{1}$, intermediate IV would be basic enough to generate more acetylide and regenerate the nucleophile from its $\beta$-ammonium acrylate resting state, and thus, keep the catalytic cycle going.

55

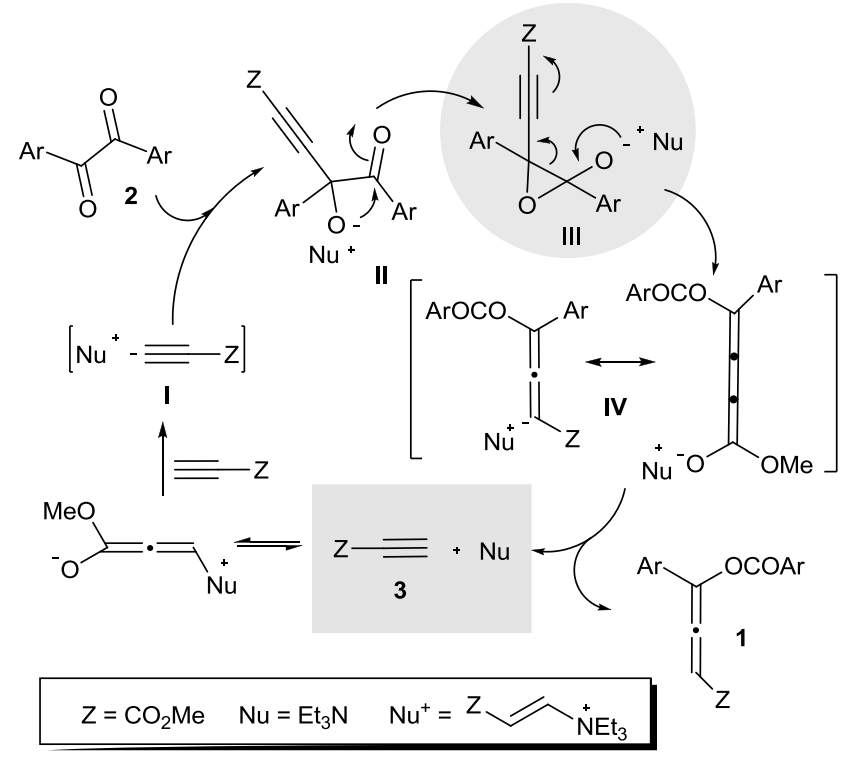

Scheme 1. Tertiary amine-catalyzed domino-based manifold

60 To test this proposal, we chose the $\mathrm{Et}_{3} \mathrm{~N}$-catalyzed reaction 
of benzil (2a) and methyl propiolate (3a) as the model reaction (Scheme 2). It was found that when a solution of both reactants in dichloromethane was treated at $0^{\circ} \mathrm{C}$ with $\mathrm{Et}_{3} \mathrm{~N}(10$ mol\%), a smooth reaction occurred affording a major product 5 which slowly transformed into other products. Interestingly, this intermediate could be detected by TLC but it could not be isolated for characterization. A more detailed study of the reaction showed that after $10 \mathrm{~min}$ at $0^{\circ} \mathrm{C}$ the practical totality of the starting benzil had been transformed into this new 10 compound. Quenching the reaction at this time with pyrrolidine afforded enamine 4 (77\%) which could be isolated and further hydrolyzed to ketone $\mathbf{5}$. Because enamine $\mathbf{4}$ is the expected addition compound on an allene such as 1aa, we assigned this structure to the main reaction intermediate, thus 15 verifying the postulated cascade of events shown in Scheme 1. This assignment was further corroborated by running the reaction in an NMR tube using $\mathrm{CDCl}_{3}$ as solvent. ${ }^{13} \mathrm{CNMR}$ analysis of the reaction mixture at different times showed that the quaternary allene peak at $210 \mathrm{ppm},{ }^{6 \mathrm{c}}$ clearly visible just 20 minutes after setting the reaction, totally disappeared when the reaction was completed. In a separate experiment, the reaction mixture was allowed to progress overnight at room temperature and under these conditions, allene 1aa was fully transformed into the mixture of cyclobutanes 6aa-7aa and 25 cyclobutene 8aa (75\%) (Scheme 2). Prolonged treatment of this mixture with $\mathrm{Et}_{3} \mathrm{~N}$ ( 1 equiv; $24 \mathrm{~h}$ ) generated the mixture of cyclobutanes 6aa and cyclobutenes 8aa via quantitative transformation of $7 \mathbf{a a}$ into 8aa. Structures of cyclobutane 6aa and cyclobutene $\mathbf{8 a a}$ were unambiguously confirmed by X-ray 30 crystallographic analyses. ${ }^{\S}$ To the best of our knowledge, this
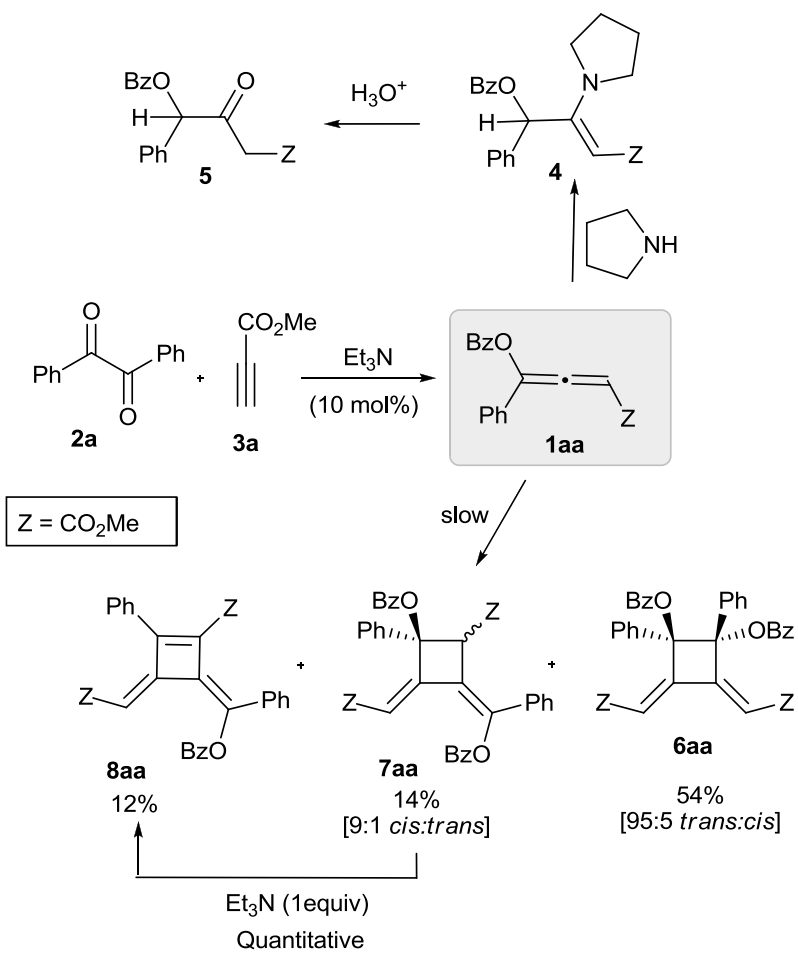

Quantitative

Scheme 2. $\mathrm{Et}_{3} \mathrm{~N}$-catalyzed reaction of benzyl and methyl propiolate.
35 protocol constitutes the first example of a synthesis of allenes involving a rearrangement of 1,2-diarylketones.

Cyclobutanes $\mathbf{6 a}$ and 7aa constitute therefore the chemical picture of the allene's basal reactivity which is expressed as a stereoselective and self-biased formal [2+2]40 cycloaddition. Due to the novelty and functional complexity of these cyclobutanic products, we undertook a study of the synthetic scope of this transformation (Table 1). The reaction proved to be general with regard to both the alkyl propiolate (entries 1-5) and the electronic nature of the aromatic 1,245 diketones (entries 1, 6-10) affording a combined yield of cyclic products 6 and 8 typically in $60-75 \%$ yield. Although relatively small amounts of 1,2-diketone starting materials were always recovered, use of excess propiolate did not increase the conversion. In all of the cases, cyclobutanes 6 50 were obtained as the major products with an impressive high trans-diastereoselectivity (more interesting $\mathrm{C}_{2}$-symmetry), while minor cyclobutenes $\mathbf{8}$ are generated as single geometric isomers. The regioselectivity of the formal [2+2]cycloaddition is moderate and it remains practically 55 unchanged in the $-78^{\circ}$ to $20^{\circ} \mathrm{C}$ temperature range. Finally and not less important for practical purposes, the reaction could be easily scaled up to $100 \mathrm{mmol}$ with a similar chemical efficiency (68\% of combined yield) (Entry 2$)$.

${ }_{60}$ Table 1. $\mathrm{Et}_{3} \mathrm{~N}$-catalyzed reaction of 1,2-diketones 2 with alkynoates 3.

\begin{tabular}{clccccccc}
\hline Entry & \multicolumn{2}{c}{$\begin{array}{c}\mathbf{2} \\
\mathrm{Ar}=\mathrm{R}-\mathrm{C}_{6} \mathrm{H}_{4^{-}}\end{array}$} & & $\mathbf{3}$ & $\mathbf{6}(\mathrm{dr})^{\mathrm{a}}$ & $\mathbf{8}$ & $\mathrm{Rsm}^{\mathrm{b}}$ \\
\hline 1 & $\mathbf{a}$ & $\mathrm{H}$ & $\mathbf{a}$ & $\mathrm{Me}$ & $51(97: 3)$ & 24 & 10 \\
2 & $\mathbf{a}$ & $\mathrm{H}$ & $\mathbf{a}$ & $\mathrm{Me}$ & $47(97: 3)^{\mathrm{c}}$ & 21 & \\
3 & $\mathbf{a}$ & $\mathrm{H}$ & $\mathbf{b}$ & $\mathrm{Et}$ & $48(96: 4)$ & 21 & 13 \\
4 & $\mathbf{a}$ & $\mathrm{H}$ & $\mathbf{c}$ & $t \mathrm{Bu}$ & $40(9: 1)$ & 28 & 23 \\
5 & $\mathbf{a}$ & $\mathrm{H}$ & $\mathbf{d}$ & $\mathrm{Bn}$ & $43(97: 3)$ & 20 & 22 \\
6 & $\mathbf{b}$ & $4-\mathrm{Me}$ & $\mathbf{a}$ & $\mathrm{Me}$ & $51(97: 3)$ & 20 & 13 \\
7 & $\mathbf{c}$ & $4-\mathrm{F}$ & $\mathbf{a}$ & $\mathbf{6}$ & $55(\geq 99)$ & 17 & 14 \\
8 & $\mathbf{d}$ & $4-\mathrm{MeO}$ & $\mathbf{a}$ & $\mathbf{6}$ & $50(97: 3)$ & 14 & 24 \\
9 & $\mathbf{e}$ & $3-\mathrm{MeO}$ & $\mathbf{a}$ & $\mathbf{6}$ & $42(96: 4)$ & 18 & 14 \\
10 & $\mathbf{f}$ & $(2-\mathrm{furylCO})_{2}$ & $\mathbf{a}$ & $\mathbf{6}$ & $22(\geq 99)$ & 6 & 20
\end{tabular}

${ }^{a}$ Trans:cis ratio determined by integration of representative ${ }^{1} \mathrm{HNMR}$ signals. ${ }^{\mathrm{b}}$ Recovered 1,2-diketone. ${ }^{\mathrm{c}} 100 \mathrm{mmol}$ scale.

65 Cyclic acenaphthenequinone $\mathbf{2 g}$ afforded spiro-1,3dioxolane derivatives 9 and a small amount of $\mathbf{6 g a}$ (Scheme 3 ). Observe that in this case, the $\mathrm{CO}-\mathrm{CO} \sigma$-bond is strong enough to inhibit the rearrangement and the transformation is funnelled toward the 1,3-dioxolane 9 formation through an ${ }_{70} \mathbf{A B B}$ ' 3CR acetylide-driven process. ${ }^{7}$ When fixing the stoichiometry to that required for the ABB' 3CR process $(2: 1)$, the yield of $\mathbf{9}$ goes up to $70 \%$ (mixture of isomers) and 6ag is not produced. 


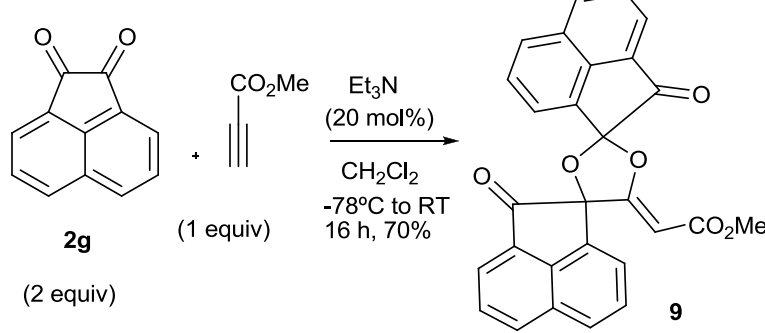

Scheme 3. Acetylide-driven 1,3-dioxolane 9 formation.

5 A preliminary DFT study at the B3LYP/6-31G* level showed a HOMO-LUMO gap of $0.17030 \mathrm{eV}$ for allene 1aa, a magnitude sufficiently small to allow an ambiphilic reactivity profile (Table 2). ${ }^{9}$ This property also explains the observed smooth tendency for dimerization and the complete observed 10 regioselectivity. Table 2 highlights the activating effect exercised by both the $\mathrm{Ph}$ and $\mathrm{OBz}$ groups allocated at $\mathrm{C}_{3}$ and the opposite effect played by the ester group at $\mathrm{C}_{1}$. Although a widely accepted stepwise mechanism involving a perpendicular bisallyl diradical ${ }^{10}$ would explain the observed 15 regio- and stereoselectivity, a concerted mechanism cannot be ruled out. ${ }^{11}$

Table 2. HOMO-LUMO energies $(\mathrm{eV})$ for $\mathrm{R}^{1} \mathrm{R}^{2} \mathrm{C}=\mathrm{C}=\mathrm{CR}^{3}$

\begin{tabular}{cccccc}
\hline $\mathbf{R}^{\mathbf{1}}$ & $\mathbf{R}^{\mathbf{2}}$ & $\mathbf{R}^{\mathbf{3}}$ & HOMO & LUMO & $\Delta \mathbf{E}$ \\
\hline $\mathrm{H}$ & $\mathrm{H}$ & $\mathrm{H}$ & -0.26297 & 0.02065 & 0.28362 \\
$\mathrm{Ph}$ & $\mathrm{H}$ & $\mathrm{H}$ & -0.21846 & -0.02670 & 0.19176 \\
$\mathrm{OBz}$ & $\mathrm{H}$ & $\mathrm{H}$ & -0.24221 & -0.05275 & 0.18946 \\
$\mathrm{H}$ & $\mathrm{H}$ & $\mathrm{CO}_{2} \mathrm{Me}$ & -0.26746 & -0.03807 & 0.22939 \\
$\mathrm{Ph}$ & $\mathrm{OBz}$ & $\mathrm{H}$ & -0.21814 & -0.04983 & 0.16831 \\
$\mathrm{Ph}$ & $\mathrm{OBz}$ & $\mathrm{CO}_{2} \mathrm{Me}$ & -0.22733 & -0.05703 & 0.17030 \\
\hline
\end{tabular}

20

In summary, we have reported a novel and efficient organocatalytic synthetic manifold to gain access to bifunctional allenes $\mathbf{1}$ from readily available starting materials, under mild reaction conditions and in very short 25 periods of times. In the absence of other chemical reactants, these allenes suffer a remarkably smooth thermally-driven dimerization to form the functionalized complex cyclobutanes $\mathbf{6}$ and cyclobutenes 8. Overall, the reaction mainly generates one $\mathrm{C}_{2}$-symmetric cyclobutane ring featuring two quaternary 30 aromatic esters, two exocyclic conjugated aliphatic esters, and thus, a $(\mathrm{Z}, \mathrm{Z})-1,3$-diene function. Interestingly, the reaction network forms two $\mathrm{C}-\mathrm{O}$ and four $\mathrm{C}-\mathrm{C}$ bonds, while only breaks two C-C bonds. Finally, and not less important, the in situ generation of these allenes is mild enough to be 35 compatible with a large number of organic functionalities and it constitutes an excellent workbench for the discovery of new complexity generating processes. The efficient formation of enamine $\mathbf{4}$ is a good example of this property. This issue is being developed in our lab.

40

Acknowledgements. This work was supported by the Spanish
Ministerio de Educación y Ciencia and the European Regional Development Fund (CTQ2005-09074-C02-02), the Spanish MSC ISCIII (RETICS RD06/0020/1046), CSIC (Proyecto ${ }_{45}$ Intramural Especial 200719) and Fundación Instituto Canario de Investigación del Cáncer (FICI-G.I.N08/2007). Authors thank technicians Sonia Rodriguez Díaz and Aida Sánchez López for preparative work.

\section{${ }_{50}$ Notes and references}

† Representative procedure: 1,2 -Diketone $2 \mathrm{a}(3.00 \mathrm{mmol})$ and methyl propiolate (3a) $(3.00 \mathrm{mmol})$ are dissolved in $\mathrm{CH}_{2} \mathrm{Cl}_{2}(5 \mathrm{~mL})$ and the solution is cooled to $0^{\circ} \mathrm{C}$ in an ice bath. $\mathrm{Et}_{3} \mathrm{~N}(0.30 \mathrm{mmol})$ is added and the reaction is allowed to react overnight without further cooling. $\mathrm{Et}_{3} \mathrm{~N}$ (3

$55 \mathrm{mmol}$ ) is added and the reaction is allowed to react for one additional day (this step simplifies the isolation of $\mathbf{6 a a}$ while transforms 7aa cis/trans into 8aa. Purification of products 6aa trans/cis and 8aa was carried out by flash column chromatography (silica gel, n-hexane/EtOAc 80/20 to 60/40). Cyclobutane 6aa trans-isomer (major): White solid, m.p. = 60 181.0-182.6 ${ }^{\circ} \mathrm{C} ;{ }^{1} \mathrm{H} \mathrm{NMR}\left(400 \mathrm{MHz}, \mathrm{CDCl}_{3}, 25^{\circ} \mathrm{C}\right): \delta=3.37(\mathrm{~s}, 6 \mathrm{H}), 6.86$ (s, 2H), 7.16-7.29 (m, 10H), $7.41(\mathrm{tt}, J=7.4$ and $1.3 \mathrm{~Hz}, 2 \mathrm{H}) 7.56(\mathrm{~m}, 4 \mathrm{H})$, 7.69 (m, 4H) $;{ }^{13} \mathrm{C}$ NMR (100 MHz, $\left.\mathrm{CDCl}_{3}, 25^{\circ} \mathrm{C}\right): \delta=\square \square 51.5,92.4$, $117.8,127.6,128.0,128.1,128.2,129.2,129.9,132.5,134.8,151.2$, 163.3, 164.7; IR $\left(\mathrm{HCCl}_{3}\right): \tilde{v}=3027.8,1730.3,1449.9,1436.1,1343.8$, 65 1268.1, 1222.3, $1108.6 \mathrm{~cm}^{-1}$; Anal. Calcd. for $\mathrm{C}_{36} \mathrm{H}_{28} \mathrm{O}_{8}: \mathrm{C}, 73.46 ; \mathrm{H}$, 4.79. Found: C, 73.47; H, 4.71; m/z (\%): $588(0.3)\left[\mathrm{M}^{+}\right], 362(8.4), 162$ (28), 130 (19), 105 (100), 77 (32). Cyclobutene 8aa: Yellow solid, m.p. = 156.5-157.9 ${ }^{\circ} \mathrm{C} ;{ }^{1} \mathrm{H}$ NMR $\left(400 \mathrm{MHz}, \mathrm{CDCl}_{3}, 25^{\circ} \mathrm{C}\right): \delta=3.32$ (s, $\left.3 \mathrm{H}\right), 3.38$ $(\mathrm{s}, 3 \mathrm{H}), 6.08(\mathrm{~s}, 1 \mathrm{H}), 7.33-7.41(\mathrm{~m}, 6 \mathrm{H}), 7.46-7.54(\mathrm{~m}, 6 \mathrm{H}), 7.65(\mathrm{tt}, J$ $70=7.5$ and $1.3 \mathrm{~Hz}, 2 \mathrm{H}), 8.19(\mathrm{~m}, 2 \mathrm{H}) ;{ }^{13} \mathrm{C} \mathrm{NMR}\left(100 \mathrm{MHz}, \mathrm{CDCl}_{3}, 25^{\circ} \mathrm{C}\right)$ : $\delta=51.0,51.5,110.4,126.5,127.5,127.9,128.0,128.6,128.77,128.79$, $129.0,129.5,130.1,131.1,133.9,134.4,136.5,142.2,149.5,156.6$, 161.5, 164.6, 165.4; IR $\left(\mathrm{HCCl}_{3}\right): v^{\sim}=3028.2,2953.0,1731.6,1435.9$, 1258.0, 1063.4 cm $\mathrm{cm}^{-1}$; Anal. Calcd. for $\mathrm{C}_{29} \mathrm{H}_{22} \mathrm{O}_{6}: \mathrm{C}, 74.67 ; \mathrm{H}, 4.75$. Found: 75 C, 74.77; H, 5.00; m/z (\%): 466 (24) [M+'], 330 (10), 215 (17), 106 (19), 105 (100), 77 (60).

$\S$ Crystal data for 6aa: $\mathrm{C}_{36} \mathrm{H}_{28} \mathrm{O}_{8}, \quad M=588.58$, monoclinic, $a=31.764(3), \quad b=10.8730(10), \quad c=18.823(2) \AA, \quad \beta=91.707(5)^{\circ}$, $V=6498.0(11) \AA^{3}, T=293 \mathrm{~K}$, space group $\mathrm{C} 2 / \mathrm{c}$ (no. 15), $Z=8$, 8034744 reflections measured, 5184 unique $\left(\mathrm{R}_{\mathrm{int}}=0.064\right)$. All nonhydrogen atoms were refined with anisotropic thermal parameters using full-matrix least squares procedures on $\mathrm{F}^{2}$. The solvent molecule (dichloromethane) presented a higher disorder and it was impossible to establish a good model even using an isotropic 85 refinement. Therefore a SQUEZZE procedure was made in order to eliminate the contribution of the solvent molecule. In any case it does not play an important role in the structure. All $\mathrm{H}$ atoms were placed in geometrically calculated positions. The methyl-H atoms were refined as rigid groups, which were allowed to rotate 90 but not to tip, with $\mathrm{U}_{\text {iso }}(\mathrm{H})=1.5 \mathrm{U}_{\text {eq }}(\mathrm{C})$. All other $\mathrm{H}$ atoms were allowed to ride on their parent atoms with $\mathrm{U}_{\text {iso }}(\mathrm{H})=1.2 \mathrm{U}_{\mathrm{eq}}(\mathrm{C})$. The final $R$ and $w R^{2}$ were 0.0770 and 0.2019 respectively. CCDC 704023. Crystal data for 8aa: $\mathrm{C}_{29} \mathrm{H}_{22} \mathrm{O}_{6}, M=466.47$, monoclinic, $a=10.769(1), \quad b=9.678(1), \quad c=23.311(1) \quad \AA, \quad \beta=96.400(2)^{\circ}$, ${ }_{95} V=2414.4(4) \AA^{3}, T=293 \mathrm{~K}$, space group $\mathrm{P} 21 / \mathrm{c}$ (no. 14), $Z=4,27643$ reflections measured, 3454 unique $\left(\mathrm{R}_{\mathrm{int}}=0.047\right)$. Only the reflections up to $0.9 \AA$ were considered in the refinement process. All non-hydrogen atoms were refined with anisotropic thermal parameters using full-matrix least squares procedures on $\mathrm{F}^{2}$. The 100 molecule presents a moderate disorder in one carboxylic group (O4-C20-03-C21) that was modeled using two sites with occupancy levels of $70 \%$ and $30 \%$ of probability. All $\mathrm{H}$ atoms were placed in geometrically calculated positions. The methyl-H atoms were refined as rigid groups, which were allowed to rotate 105 but not to tip, with $\mathrm{U}_{\text {iso }}(\mathrm{H})=1.5 \mathrm{U}_{\mathrm{eq}}(\mathrm{C})$. All other $\mathrm{H}$ atoms were 
allowed to ride on their parent atoms with $\mathrm{U}_{\text {iso }}(\mathrm{H})=1.2 \mathrm{U}_{\mathrm{eq}}(\mathrm{C})$. The final $R$ and $w R^{2}$ were 0.0699 and 0.1955 respectively. CCDC 704024.

5 1. A. K. Yudin and R. Hili, Chem. Eur. J. 2007, 13, 6538.

2. A. Dömling, Chem Rev. 2006, 106, 17.

3. (a) Modern Allene Chemistry, eds. N. Krause and A.S.E.K. Hashmi, Wiley-VCH: Weinheim, 2004; (b) K. M. Brummond and J. E. DeForrest, Synthesis 2007, 795; (c) S. Ma, Chem. Rev. 2005, 105, $10 \quad 2829$.

4. For a selected review: D. J. Pasto, Tetrahedron 1984, 40, 2805.

5. (a) D. Tejedor, S. López-Tosco, F. Cruz-Acosta, G. Méndez-Abt and F. García-Tellado, Angew. Chem. Int. Ed. 2008, DOI: 10.1002/anie.200801987; (b) D. Tejedor, D. González-Cruz, A.

15 Santos-Expósito, J. J. Marrero-Tellado, P. de Armas and F. GarcíaTellado, Chem. Eur. J. 2005, 11, 3502.

6. The number of reported examples in the bibliography for these units is scarce. For selected examples, see: (a) T. Schwier, A. W. Sromek, D. M. L. Yap, D. Chernyak and V. Gevorgyan, J. Am. Chem. Soc. 20 2007, 129, 9868 and references cited therein; (b) C. Bee and M. A. Tius, Org. Lett. 2003, 5, 1681; (c) M. R. Sestrick, M. Miller and L. S. Hegedus, J. Am. Chem. Soc. 1992, 114, 4079.

7. (a) D. Tejedor, A. Santos-Expósito and F. García-Tellado, Chem. Eur. J. 2007, 13, 1201; (b) D. Gonzalez-Cruz, D. Tejedor, P. de

25 Armas and F. García-Tellado, Chem. Eur. J. 2007, 13, 4823;(c) D. Tejedor, F. García-Tellado, J. J. Marrero-Tellado and P. de Armas, Chem. Eur. J. 2003, 9, 3122.

8. For a precedent for this rearrangement: A. N. Pillai, C. H. Suresh, C. H. and V. Nair, Chem. Eur. J. 2008, 14, 5851.

30 9. (a) A. Rauk, in Orbital Interaction Theory of Organic Chemistry, John Wiley and Sons, 1994, pp 118; (b) D. Nori-Shargh, F. Deyhimi, J. E. Boggs, J.-B Saeed and R. Shakibazadeh, J. Phys. Org. Chem., $2007,20,355$.

10. a) For an interesting example of allene dimerization involving stabilized bisallyl biradical intermediates see: E. V. Banide, Y. Ortin,, C. M. Seward, L. E. Harrington, H. Müller-Bunz and M. J. McGlinchey, Chem. Eur. J. 2006, 12, 3275 and references cited therein; (b) for mechanistic considerations on the biradical mechanism, see: M. Christl, S. Groetsch and K. Günter, Angew. Chem. Int. Ed. 2000, 39, 3261 and reference cited therein.

11. A theoretical study of this reaction is undergoing in our group. 